\title{
Experimental analyses of the source of ABR wave II
}

\author{
C. Tait*, J. Miller, Y. Cycowicz, and H. Sohmer \\ Department of Physiology, Hebrew University-Hadassah Medical School, P.O.B. 1172, Jerusalem 91010, Israel
}

Summary. Recent reports based on direct recording from various locations along the auditory nerve in humans during neurosurgical procedures have suggested that the auditory nerve-brainstem response (ABR) wave II is generated by the same neurons which generate wave I. In order to analyze this possibility using a different approach, ABR was recorded in ten rats in response to several click intensities and click repetition rates. These studies were also repeated in ten human volunteers. The amplitudes and latencies of ABR waves I, II and III were analyzed in order to determine if wave I and II behaved in a parallel fashion with changes in stimulus intensity and latency, as would be expected from the physiological "all or none law" if both waves were generated by the same axons. Several types of analyses indicate that the amplitudes of ABR waves I and II do not grow in amplitude in a parallel fashion with increases in stimulus intensity and decreases in stimulus rate. This is evidence either for independent sources of waves I and II or for composite sources of wave II both from the auditory nerve and the cochlear nuclei.

Key words: Auditory nerve - Brainstem - Evoked response - Source

\section{Introduction}

The auditory nerve-brainstem response (ABR) waveform is considered to reflect the far-field volume conducted potentials which are generated in the auditory nerve and the ascending brainstem auditory

\footnotetext{
* Permanent address: Speech and Language Pathology, School of Education, University of Michigan, Ann Arbor, Michigan, USA

Offprint requests to: $\mathrm{H}$. Sohmer
}

pathway. These potentials have been shown to be invaluable in hearing screening in infants and in difficult-to-test children $[12,13]$, as well as in the neurological diagnosis of brainstem lesions $[14,16]$.

An understanding of the origin of the $\mathrm{ABR}$ wave components can be of great importance in the clinical application of ABR, and therefore has been of significant interest to a number of investigators. There is a general consensus among researchers that the generating sites of waves I, III, IV, and V are, respectively, the auditory nerve, the superior olivary complex and the region of the lateral lemniscus-inferior colliculus. However, controversy and uncertainty have recently been introduced regarding the origin of scalprecorded wave II in humans following experiments involving the introduction of direct intracranial recordings in patients during neurosurgical procedures that have included direct recordings from the auditory nerve and its vicinity [1,2, 5-9]. These recordings were made from proximal and distal portions of the eighth nerve, from the entrance of the eighth nerve into the brainstem, and from additional brainstem sites.

Moller and his group [7] have concluded from direct $\mathrm{ABR}$ recordings that the sole source of wave II is the auditory nerve as it exits the internal auditory meatus. They have found additional theoretical support for this from considerations of the length of the auditory nerve, the nerve conduction velocity and synaptic delay. These findings have led them to conclude that the second-order neurons (cochlear nucleus) would be expected to fire at times coinciding with the appearance of ABR wave III [9]. Based on essentially similar direct recordings [2] and theoretical evidence [1], Hashimoto and his group concluded that wave II is generated by neurons from the cochlear nucleus as well as from some secondary 
Table 1. Regression analysis for the dependence of the latency and amplitude of ABR waves I, II and III on click intensity and rate in humans and in rats

\begin{tabular}{|c|c|c|c|c|c|c|c|c|c|}
\hline Subjects & Function & Parameter & Wave & $r$ & $P<$ & Slope & & $\mathrm{SE}$ & Intercept \\
\hline \multirow[t]{12}{*}{ Humans } & \multirow[t]{6}{*}{ Intensity (dB) } & \multirow[t]{3}{*}{ Latency (ms) } & I & -0.83 & 0.001 & -0.0014 & \multirow{3}{*}{$\mathrm{D}$} & 0.001 & 2.72 \\
\hline & & & II & -0.58 & 0.001 & -0.0063 & & 0.001 & 3.34 \\
\hline & & & III & -0.66 & 0.001 & -0.0072 & & 0.001 & 4.33 \\
\hline & & \multirow[t]{3}{*}{ Amplitude $(\mu \mathrm{V})$} & I & 0.62 & 0.001 & 0.0509 & & 0.009 & -3.64 \\
\hline & & & II & -0.03 & NS & - & & - & - \\
\hline & & & III & 0.79 & 0.001 & 0.0586 & & 0.007 & -4.42 \\
\hline & \multirow[t]{6}{*}{ Rate (clicks/s) } & \multirow[t]{3}{*}{ Latency (ms) } & $I$ & 0.36 & 0.025 & 0.0020 & \multirow{3}{*}{$\mathrm{S}$} & 0.0001 & 1.34 \\
\hline & & & II & 0.49 & 0.005 & 0.0022 & & 0.001 & 2.53 \\
\hline & & & III & 0.45 & 0.005 & 0.0016 & & 0.001 & 3.43 \\
\hline & & \multirow[t]{3}{*}{ Amplitude $(\mu \mathrm{V})$} & I & -0.67 & 0.001 & -0.0221 & \multirow{3}{*}{$\mathrm{D}$} & 0.004 & 2.98 \\
\hline & & & II & -0.48 & 0.005 & -0.0090 & & 0.003 & 1.19 \\
\hline & & & III & -0.42 & 0.001 & -0.0108 & & 0.004 & 2.75 \\
\hline \multirow[t]{12}{*}{ Rats } & \multirow[t]{6}{*}{ Intensity (dB) } & \multirow[t]{3}{*}{ Latency (ms) } & I & -0.63 & 0.001 & -0.0035 & \multirow{3}{*}{$\mathrm{D}$} & 0.001 & 1.27 \\
\hline & & & II & -0.68 & 0.001 & -0.0678 & & 0.001 & 2.56 \\
\hline & & & III & -0.41 & 0.005 & -0.0033 & & 0.001 & 2.90 \\
\hline & & \multirow[t]{3}{*}{ Amplitude $(\mu \mathrm{V})$} & I & 0.62 & 0.001 & 0.0340 & \multirow{3}{*}{$S$} & 0.006 & -2.02 \\
\hline & & & II & -0.61 & 0.001 & 0.0314 & & 0.006 & -2.05 \\
\hline & & & III & 0.60 & 0.001 & 0.0105 & & 0.002 & 0.89 \\
\hline & \multirow[t]{6}{*}{ Rate (clicks/s) } & \multirow[t]{3}{*}{ Latency (ms) } & I & 0.09 & NS & - & & - & - \\
\hline & & & II & 0.21 & NS & - & & - & - \\
\hline & & & III & 0.13 & NS & - & & - & - \\
\hline & & \multirow[t]{3}{*}{ Amplitude $(\mu \mathrm{V})$} & I & -0.26 & NS & - & & - & - \\
\hline & & & II & -0.32 & 0.05 & -0.0064 & & 0.003 & 1.46 \\
\hline & & & III & -0.21 & NS & - & & - & - \\
\hline
\end{tabular}

$r=$ correlation coefficient $P<=$ its significance; $\mathrm{SE}=$ standard error of the slope; $\mathrm{S}=$ the slopes are similar; $\mathrm{D}=$ the slopes are different

axon volleys of the eighth nerve. Thus, even though both groups employed similar techniques and considerations, quite opposite conclusions were reached.

In a separate series of experiments, Moller [4] also conducted direct recordings from the auditory nerve in rats. His results and considerations of the shorter length of the auditory nerve in the rat have led him to conclude that wave II in the rat is generated by neurons of the cochlear nucleus.

In this study, the physiological "all or none law" has been applied to obtain physiological evidence concerning the source of the ABR wave II. Moller et al. [7] concluded that wave I is the compound action potential of the auditory nerve fiber impulses in the cochlea (i.e., in the distal portion of the nerve), while wave II is the compound action potential generated by the same impulses as they exit the internal meatus (proximal portion of the nerve). Thus, if waves I and II truly reflect the same impulses that are coming from different portions of the nerve, the second wave
- according to the "all or none law" - should behave exactly parallel to that of the first wave in stimulus intensity and rate studies. The present investigation was therefore devised to compare the slopes of the amplitudes and latencies of waves I, II and III in intensity and rate functions in humans and in rats.

\section{Materials and methods}

This study was conducted on ten normal hearing volunteers and ten white Sabra laboratory rats. The human subjects were taken from a larger group of volunteers (aged 15-25 years) and were selected for having clear and large amplitude wave II over a wide range of click intensities and stimulus repetition rates. In addition to having normal hearing, all subjects were free of any known neurological disorders.

The ABR recordings in all animal and clinical studies were obtained from one ear, using a Microshev 2000 ABR system with alternate polarity clicks of various intensities and repetition rates. The electrical activity was recorded in our volun- 
teers as the potential difference between ipsilateral earlobe and scalp vertex electrodes (ground at the contralateral earlobe) and needle electrodes attached to the vertex and ipsilateral paw (ground at the tail) in the rats studied. The recorded electrical activity was filtered $(200-2000 \mathrm{~Hz})$, amplified (about $10^{6}$ ), and averaged. There were approximately 1000 stimulus repetitions for the volunteers and 128 repetitions for the rats. Two replications of each recording were made.

The rats were anesthetized with pentobarbital IP. Normal body temperature was maintained with the use of the electric surface heat. The earphone was clamped at a constant distance from each animal's ear (just above the pinna). Auditory brainstem recordings in both humans and rats were made in response to $135,125,115,105,95 \mathrm{~dB}$ peSPL clicks $(95,85,75,65$ and $55 \mathrm{~dB}$ HL respectively), at stimulus repetition rates of $20 / \mathrm{s}$, and to $115 \mathrm{~dB}$ SPL ( $75 \mathrm{~dB} \mathrm{HL})$ at stimulus repetition rates of $10 / \mathrm{s}, 20 / \mathrm{s}, 40 / \mathrm{s}$ and $80 / \mathrm{s}$. Measurements of absolute latencies and peak-to-peak amplitudes of waves I, II, III were made.

The data were subjected to linear correlation and regression analyses to determine whether or not the amplitudes (and latencies) of wave II paralleled those of wave I as a function of the click intensity and of the click repetition rate. For comparison, the slope of wave III was also determined as a function of these stimulus parameters, since there is general agreement that wave III is a postsynaptic event.

\section{Results}

Table 1 shows the correlation and regression parameters for ABR waves I-III. Regression parameters were not calculated for those cases not showing significant correlation coefficients (for example the dependence of wave I, II, III latency on click repetition rates in rats). The slopes of the regression lines for wave II were compared to those for wave I whenever possible by considering the standard error for the slopes and the chances for possible overlap.

Accordingly, we found that the slope of the dependency of wave II latency on click intensity is significantly different from that of wave I in our volunteers. In contrast, the dependence of wave II latency on click rate was similar to that of wave I. Further, the slope of wave II amplitude with click rate was different from that of wave I in our volunteers. Thus, even though there is a strong correlation between the amplitude of wave I and click intensity, there is no correlation of wave II amplitude with intensity.

In the rat intensity functions, the slope of the latency of wave II differed from that of wave I while the slopes of the amplitudes recorded were similar.

An additional analysis in our volunteers showed that the average slope of the dependence of the amplitude difference between waves II and I on click intensity differed significantly from 0 . This also indicates that the amplitude of these two waves does not grow in parallel with intensity. Other types of analyses gave similar results.

\section{Discussion}

This study has been an indirect attempt to determine the source of wave II in humans and in rats by studying the slope of the amplitude and latency of this wave as a function of stimulus intensity and repetition rate. The physiological "all or none law" provides the theoretical basis for such a study. Moller et al. [7] have maintained that wave I in humans represents the compound action potential of eighth nerve impulses from the distal segments of the nerve fibers in the cochlea before they enter the internal meatus, while wave II is the compound action potential from the same impulses generated in the same nerve fibers as they exit the internal auditory meatus. Under these circumstances, waves I and II should behave similarly in intensity and rate functions. In other words, once a nerve fiber fires an impulse in the cochlea and contributes to wave $I$, it is inconceivable that this impulse should not be propagated along the same axon and contribute to wave II if this second wave were indeed generated by the same fibers that generate wave I, as maintained by Moller et al. [7]. Therefore, as stimulus intensity is elevated or rate is decreased, the increasing number of nerve fibers responding should contribute in an equal (parallel) fashion to both waves I and II. Since a synapse is not necessarily a one-to-one impulse relay, if a synapse were to intervene between the generators of waves I and II, then wave II may not parallel wave I as the stimulus rate or intensity is varied. These considerations are more applicable to possible parallel changes in amplitudes rather than the latencies of waves I and II, since interpeak latencies are relatively invariable with changes in stimulus intensity and rate.

We have found that the dependence of the amplitude of wave II on intensity in our human subjects was not significant, while that for wave I was highly significant. These findings should be considered as evidence that these two waves do not grow in amplitude with intensity in a parallel fashion. Other types of analyses show that the amplitudes of waves I and II do not grow in parallel. The dissimilarity of the slopes of the rate functions for amplitude of waves I and II could also lead to the conclusion that they are generated by different neurons, with a synapse intervening. Since the slope of the amplitude - intensity function of wave II in the rat is similar to that of wave I, one could conclude that they are both generated by the same neurons.

Our study was designed to attempt to corroborate, although in an indirect fashion, the conclusions of Moller et al. [5-9] that both waves I and II are generated by the same neurons. Even though this conclusion was reached from direct clinical record- 
ings in patients during neurosurgical procedures, it is in need of further support, particularly since the intracranial recordings used could not always be directly compared with scalp recordings. This is due to the fact that the two recording techniques were not always conducted concurrently and different analog filters, different acoustic transducers and different scalp electrode sites were used $[6,7]$.

Our findings might also be supported by studies using multiple electrode sites with reference to noncephalic electrodes. However, the conclusions from such studies also conflict. While several scalp distribution studies conclude that waves I and II have different polarities and different scalp distributions $[3,10,15]$, the results of similar studies as well as considerations of eighth nerve conduction velocity and length indicate that wave II is generated by the same nerve fibers as wave I [11], in agreement with Moller et al. [7]. This consideration also leads to the assignment of wave III to the cochlear nucleus $[7,11]$.

Thus, at the present time direct nerve recordings $[1,2,5-9]$, scalp distribution studies $[3,10,11,15]$ and theoretical considerations $[2,9,11]$ have led to conflicting conclusions concerning the origins of several of the $A B R$ waves. In contrast, the results of our study using other physiological considerations and experiments are more compatible with the hypothesis that waves I and II are generated by independent sources (i.e., the auditory nerve and cochlear nucleus, respectively) or that wave II is a composite of simultaneous components from the auditory nerve and the cochlear nucleus, as suggested by Hashimoto et al. $[1,2]$.

Acknowledgement. The authors thank Ms L. Deutsch for her assistance in conducting the recordings.

\section{References}

1. Hashimoto I (1982) Auditory evoked potentials recorded directly from the human VIIIth nerve and brain stem: origins of their fast and slow components. In: Buser PA, Cobb WA, Okuma T (eds) Kyoto Symposia (EEG Suppl No 36). Elsevier, Amsterdam, pp 305-314
2. Hashimoto I, Ishiyama Y, Yoshimoto T, Nemoto S (1981) Brain stem auditory-evoked potentials recorded directly from human brain stem and thalamus. Brain 104:841-859

3. Hashimoto I, Ishiyama Y, Manaka S, Ebe M, Sano K (1981) Spatial distribution of brainstem auditory evoked potentials and their alterations in lesions of the VIIIth nerve and brainstem. Neurol Res 3:167-194

4. Moller AR (1983) On the origin of the compound action potentials $(\mathrm{N} 1, \mathrm{~N} 2)$ of the cochlea of the rat. Exp Neurol $80: 633-644$

5. Moller AR, Jannetta PJ (1982) Auditory evoked potentials recorded intracranially from the brainstem in man. Exp Neurol 78:144-157

6. Moller AR, Jannetta PJ (1982) Comparison between intracranially recorded potentials from the human auditory nerve and scalp recorded auditory brainstem response (ABR). Scand Audiol 11:33-40

7. Moller AR, Jannetta P, Bennett M, Moller MB (1981) Intracranially recorded responses from the human auditory nerve: new insights into the origin of brain stem evoked potentials (BSEPs). Electroencephalogr Clin Neurophysiol $52: 18-27$

8. Moller AR, Jannetta PJ, Moller MB (1981) Neural generators of brainstem evoked potentials. Results from human intracranial recordings. Ann Otol 90:591-596

9. Moller AR, Jannetta P, Moller MB (1982) Intracranially recorded auditory nerve response in man. Arch Otolaryngol 108:77-82

10. Picton TW, Hillyard SA, Krausz HI, Galambos R (1974) Human auditory evoked potentials. I. Evaluation of components. Electroencephalogr Clin Neurophysiol 36:179190

11. Scherg M, von Cramon D (1985) A new interpretation of the generators of BAEP waves I-V: results of a spatiotemporal dipole model. Electroencephalogr Clin Neurophysiol 62:290-299

12. Schulman-Galambos C, Galambos R (1975) Brainstem auditory evoked responses in premature infants. J Speech Hear Res 18:456-465

13. Sohmer H, Feinmesser M (1973) Routine use of electrocochleography (cochlear audiometry) on human subjects. Audiology 12:167-173

14. Starr A, Achor LJ (1975) Auditory brainstem responses in neurological disease. Arch Neurol 32:761-768

15. Starr A, Squires K (1982) Distribution of auditory nerve brainstem potentials over the scalp and nasopharynx in humans. Ann NY Acad Sci 388:427-442

16. Stockard JJ, Rossiter VS (1977) Clinical and pathologic correlates of brainstem auditory response abnormalities. Neurology $27: 316-325$

Received October 31, 1986 / Accepted November 9, 1986 